\title{
Le poisson zèbre (danio rerio), un modèle en biologie du développement
}

Le poisson zèbre danio rerio constitue un modèle privilégié pour l'étude du développement des vertébrés. Les embryons sont facilement accessibles, peuvent être produits en grand nombre et leur développement est facile à observer grâce à leur transparence. Le temps de génération est très bref, toutes les caractéristiques du poisson adulte étant acquises au $7 \mathrm{e}$ jour. L'irradiation aux rayons $\gamma$ est un moyen commode d'obtention de mutations dont les effets sont aisés à observer grâce aux méthodes permettant d'obtenir des individus haploïdes ou des diploïdes homozygotes par gynogenèse. La transgenèse est enfin possible dans cette espèce. Une limitation actuelle à ce système est constituée par le caractère rudimentaire de sa cartographie génétique ; cette insuffisance est en voie d'être comblée.

\section{Marc Ekker}

Marie-Andrée

\section{Akimenko}

\section{ADRESSE}

M. Ekker : associé de recherche. M.-A. Akimenko : chargée de recherche à l'Institut Pasteur. Institute of neuroscience, University of Oregon, Eugene, OR 97403, États-Unis.

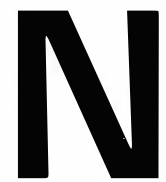

os connaissances dans le domaine de la biologie du développement ont beaucoup progressé au cours de la dernière décennie, particulièrement grâce aux études faites sur des invertébrés tels la mouche Drosophila melanogaster et le ver Caenorhabditis elegans. Ces deux organismes se prêtent aisément à une combinaison d'analyses embryologiques et génétiques. Leur développement est facile à observer ; on peut créer des mutations chez l'embryon et étudier les perturbations qu'elles entraînent dans le développement. Chez les vertébrés, un modèle expérimental offrant les avantages de la drosophile ou de $C$. elegans serait un atout précieux pour l'étude des mécanismes de développement qui leur sont spécifiques. Malheureusement, un tel modèle n'existe pas encore. Le développement de la souris et celui de la grenouille, Xenopus laevis, ont été étudiés de manière approfondie, mais la croissance in utero de l'embryon de souris le rend difficilement accessible et le temps de génération du xénope est trop long pour permettre une approche génétique facile. Des études récentes effectuées sur l'embryon du poisson zèbre danio rerio (Brachydanio rerio, zebrafish) indiquent que ce petit poisson bien connu des aquariophiles offre la possibilité d'une approche embryologique tout en pouvant se prêter à des études génétiques et pourrait devenir ainsi l'équivalent de la drosophile chez les vertébrés.

L'élevage du danio est facile et requiert relativement peu d'espace, chaque adulte ne mesurant que trois centimètres. A chaque croisement, quelques centaines d'œufs sont produits et un croisement peut être répété à quelques jours d'intervalle. Les œufs fécondés se développent de manière synchrone à l'extérieur du corps de la mère et sont ainsi disponibles pour l'observation à tous les 


\section{RÉFÉRENCES}

1. Kuwada JY. Cell recognition by neuronal growth cones in a simple vertebrate embryo. Science $1986 ; 233: 740-6$.

2. Kimmel CB, Warga RM. Tissue-specific cell lineages originate in the gastrula of the zebrafish. Science 1986; 231 : 365-8.

3. Kimmel CB, Warga RM, Schilling TF. Origin and organization of the zebrafish fate map. Development 1990 ; 108 : 581-94.

4. Dale L, Slack JMW. Regional specification within the mesoderm of early embryos of Xenopus laevis. Development 1987 ; $100: 279-95$.

5. Kimmel CB, Westerfield M. Signals and Sense: Local and Global Order in Perceptual Maps. New York : John Wiley and Sons, 1991 (sous presse).

6. Wilson SW, Ross LS, Parrett T, Easter Jr Ss. The development of a simple scaffold of axon tracts in the brain of the embryonic zebrafish, Brachydanio rerio. Development $1990 ; 108: 121-45$.

7. Grunwald DJ, Kimmel CB, Westerfield M, Walker C, Streisinger G. A neural degeneration mutation that spares primary neurons in the zebrafish. Dev Biol 1988 ; $126: 115-28$.

8. Eisen JS, Myers PZ, Westerfield $M$. Pathway selection by growth cones of identified motoneurons in live zebrafish embryos. Nature 1986 ; 320 : 269-71.

9. Westerfield M, Eisen JS. Neuromuscular specificity: pathfinding by identified motor growth cones in a vertebrate embryo. Trends Neurosci 1988; 11: 18-22.

10. Eisen JS, Pike SH, Debu B. The growth cones of identified motoneurons in embryonic zebrafish select appropriate pathways in the absence of specific cellular interactions. Neuron 1989; 2 : 1097-1104.

11. Pike SH, Eisen JS. Interactions between identified motoneurons in embryonic zebrafish are not required for normal motoneuron development. J Neurosci $1990 ; 10$ : stades du développement. Les embryons sont presque totalement transparents, ce qui permet la visualisation des cellules sans avoir à disséquer l'embryon. Les propriétés optiques de l'embryon de danio se retrouvent chez d'autres téléostéens, tel le médaka japonais [1], et découlent de la ségrégation du vitellus en une cellule géante à la surface de laquelle se développe l'embryon (position télolécitale du vitellus ; figure 1). Le développement du danio est rapide ; la plupart des caractéristiques des poissons adultes peuvent être observées sept jours après la fécondation, alors que les embryons ne mesurent que quelques millimètres, ce qui rend possible la recherche de mutants parmi un grand nombre.

Quelques exemples d'études traitant de la différenciation cellulaire et de la formation du système nerveux central chez le danio seront présentés ici. Ces études ont été réalisées en majeure partie à l'université de l'Oregon, dans les laboratoires de Judith Eisen, Charles Kimmel et Monte Westerfield. Nous décrirons ensuite quelques techniques qui permettent la manipulation génétique de l'embryon de danio et nous montrerons comment les approches embryologiques et génétiques sont complémentaires.

\section{L'origine des cellules différenciées chez le danio}

Chez les organismes multicellulaires, les cellules embryonnaires dérivent de l'œuf fécondé par une série de divi-
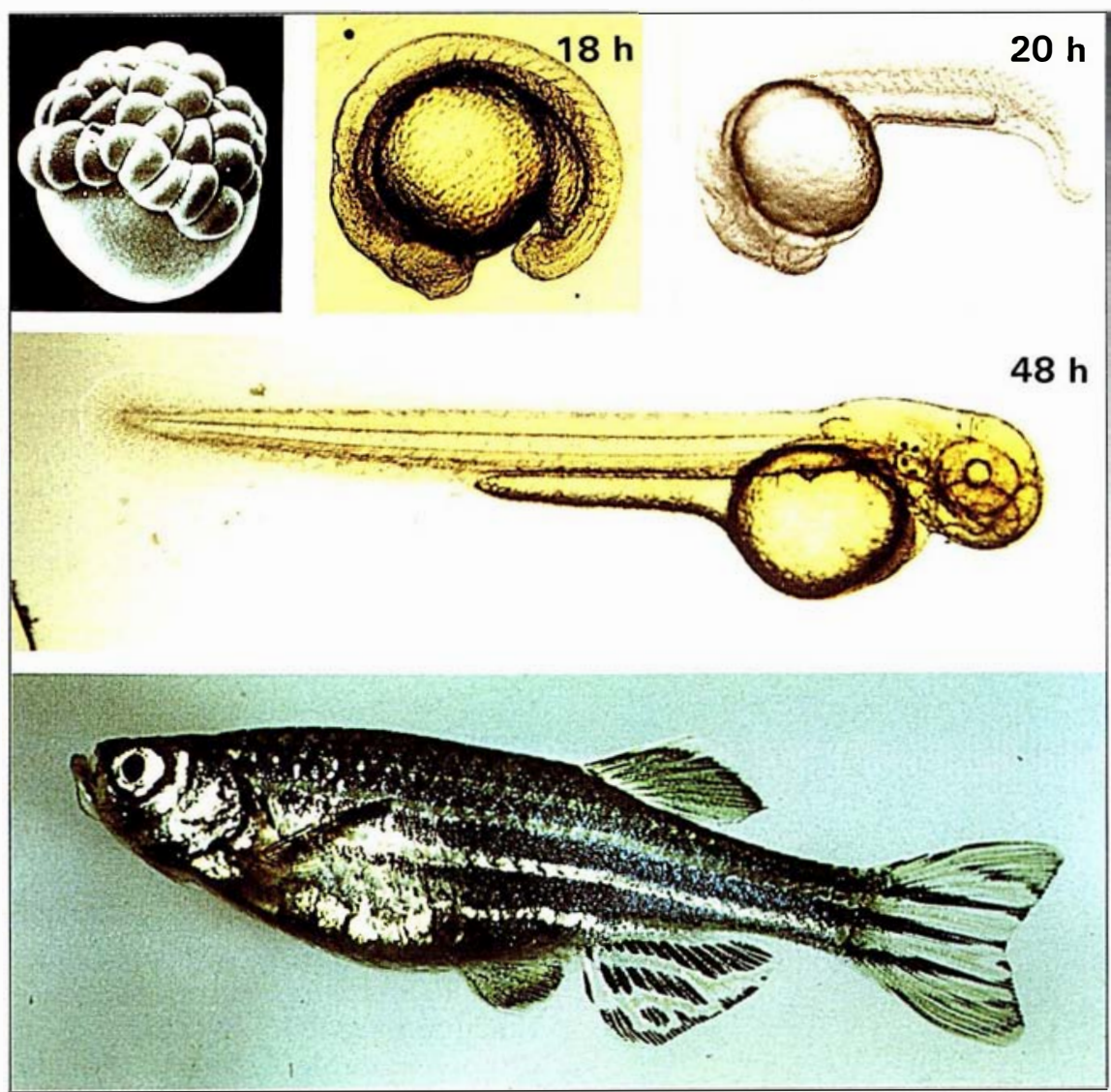

Figure 1. Le développement du danio rerio. A partir du haut et de gauche à droite, on peut voir un œuf fécondé qui a commencé à se diviser (remarquer les cellules à la surface du vitellus) ; l'embryon du danio est transparent 18 heures après la fécondation, il fait alors $1,5 \mathrm{~mm}$ de longueur ; à 20 heures, on voit clairement la segmentation de la musculature du tronc; la taille de l'embryon atteint $4 \mathrm{~mm}$ à 48 heures, ce qui représente 70 fois le diamètre de l'œuf. En bas, un danio adulte qui mesure $25 \mathrm{~mm}$. 
sions qui produiront par la suite des cellules dont le potentiel développemental est restreint. Pour certains animaux, comme par exemple le ver Caenorhabditis elegans, le potentiel développemental d'une cellule, que l'on peut définir comme étant l'ensemble des types de cellules différenciées que l'on retrouvera parmi ses descendantes, est dicté uniquement par son lignage. En revanche, pour d'autres animaux tels que la mouche, Drosophila melanogaster, la position et les interactions cellulaires influenceront le potentiel de développement d'une cellule et celle-ci pourra transmettre les restrictions ainsi établies à ses descendantes. Le potentiel de développement des cellules embryonnaires a été relativement peu étudié chez les vertébrés, partiellement à cause de leur plus grande complexité et de l'absence d'un modèle expérimental permettant une approche génétique aisée de l'ontogenèse.

Aux yeux de Charles Kimmel et de ses collaborateurs, le danio est apparu comme étant un organisme particulièrement bien adapté à l'étude du lignage cellulaire chez les vertébrés. Ils ont voulu déterminer si le destin d'une cellule embryonnaire du danio est dicté par son lignage ou par sa position. La transparence de l'embryon du danio et son développement rapide ont permis de visualiser et de suivre les cellules à partir d'un stade précoce par des techniques de traçage. Un colorant fluorescent non toxique marque les cellules, est transmis aux cellules de leur descendance et permet de suivre leurs migrations durant toute la durée de l'embryogenèse.

Les premières divisions cellulaires donnent naissance à un arrangement stéréotypique de cellules appelées blastomères. Kimmel et Warga ont marqué des blastomères issus de la même série de divisions dans des embryons différents et ont ainsi montré que la descendance d'un blastomère donné est constituée d'ensembles différents de types cellulaires qui sont localisés à des positions distinctes d'un embryon à l'autre [2]. Cela indique que la destinée d'une cellule n'est pas uniquement dictée par le lignage dont elle est issue. Si la destinée d'une cellule de l'embryon précoce est indéterminée, certaines restrictions apparaissent ultérieurement quant aux types cellulaires auxquels les descendants de cette cellule pourront contribuer. Ainsi, au stade de la gastrulation, au cours duquel les cellules migrent et réarrangent leur position dans l'embryon, d'importantes restrictions du destin des descendants d'une cellule sont établies [3]. Toujours grâce aux techniques de traçage, Kimmel et ses collaborateurs ont cartographié la destinée des descendants d'une cellule selon la position que celle-ci occupe au début de la gastrulation. Cette carte ressemble beaucoup à celle établie pour les amphibiens (figure 2) [3, 4]. Il semble donc que le destin ultime d'une cellule dépende non pas de ses ancêtres mais du milieu dont elle est issue. La question du rôle que jouent la position et les antécédents génétiques sur la destinée d'une cellule se pose également lors de la formation du système nerveux. A ce problème s'ajoute alors celui de la spécificité

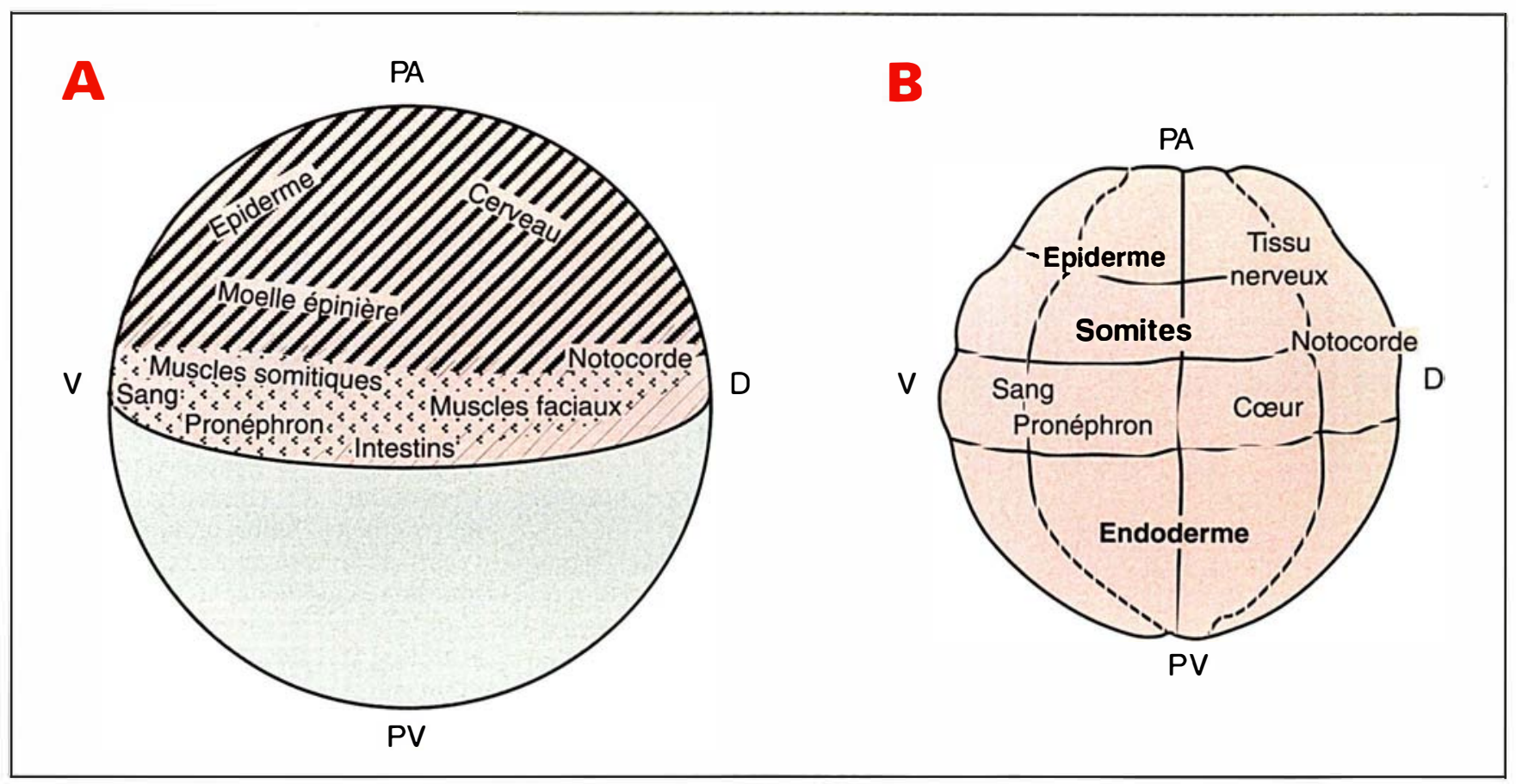

Figure 2. Carte de la destinée des cellules du danio (A) au début de la gastrulation [3] et du xénope (B) au stade de 32 cellules [4]. Ces cartes donnent un aperçu global du potentiel de développement des cellules embryonnaires. Les frontières délimitant les diverses destinées cellulaires ne sont pas indiquées et sont, en fait, plus diffuses que ne le laissent entendre les représentations. On pourra néanmoins remarquer le fort degré de similitude entre les cartes établies pour les deux espèces. $V$ : face ventrale ; $D$ : face dorsale ; PA : pôle animal ; PV : pôle végétal. (Adaptée de [3].) 


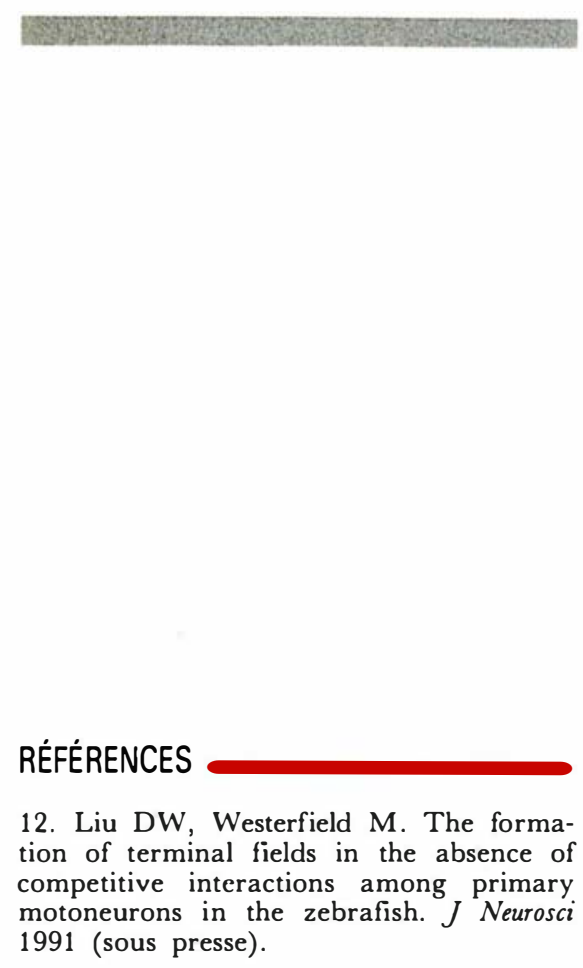

13. Streisinger G, Walker C, Dower N, Knauber D, Singer F. Production of clones of homozygous diploid zebrafish (Brachydanio rerio). Nature 1981; 291 : 293-6.

14. Streisinger G. Attainment of minimal biological variability and measurements of genotoxicity: production of homozygous diploid zebrafish. Natl Cancer Inst Monogr $1984 ; 65: 53-8$

15. Streisinger G, Singer F, Walker C, Kanauber D, Dower N. Segregation analyses and gene-centromere distances in zebrafish Genetics 1986; 112: 311-9.

16. Felsenfeld AL, Walker C, Westerfield M, Kimmel C, Streisinger G. Mutations affecting skeletal myofibril structure in the zebrafish. Development 1990 ; 108 : 443-59.

17. Kimmel CB, Kane DA, Walker C, Warga RM, Rothman MB. A mutation that changes cell movement and cell fate in the zebrafish embryo. Nature $1989 ; 337$ : $358-62$.

18. Ho RK, Kane DA. Cell-autonomous action of zebrafish spt-1 mutation in mesodermal precursors. Nature 1990 ; 348 : 728-30

19. Debu B, Pike SH, Bremiller R, Kimmel CB, Eisen JS. A small subpopulation of myocytes may be required for proper morphogenesis of identified motoneurons in embryonic zebrafish. Soc Neurosci Abstr $1989 ; 15: 877$.

20. Fjose A, Eiken HG, Njølstad PR, Molven $A$, Hordvik I. A zebrafish engrailedlike homeobox sequence expressed during embryogenesis. FEBS Lett $1988 ; 231$
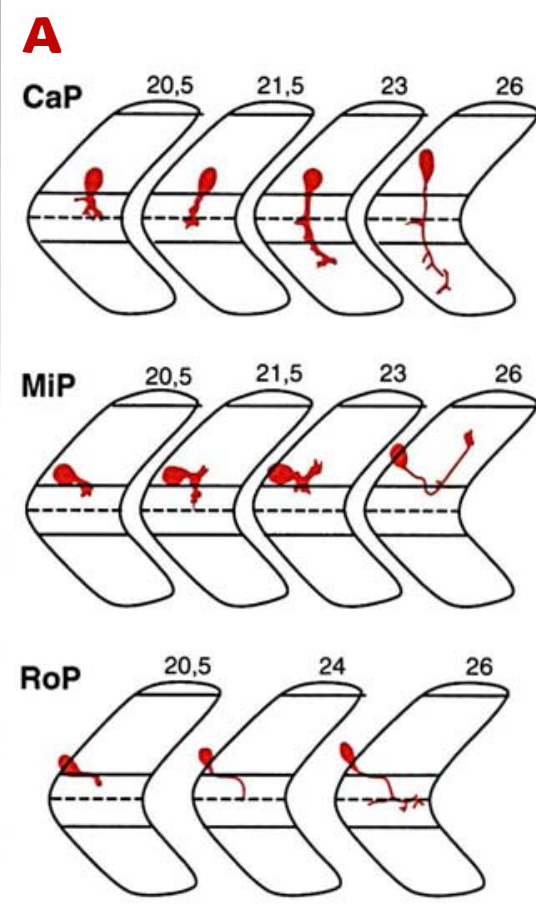

B

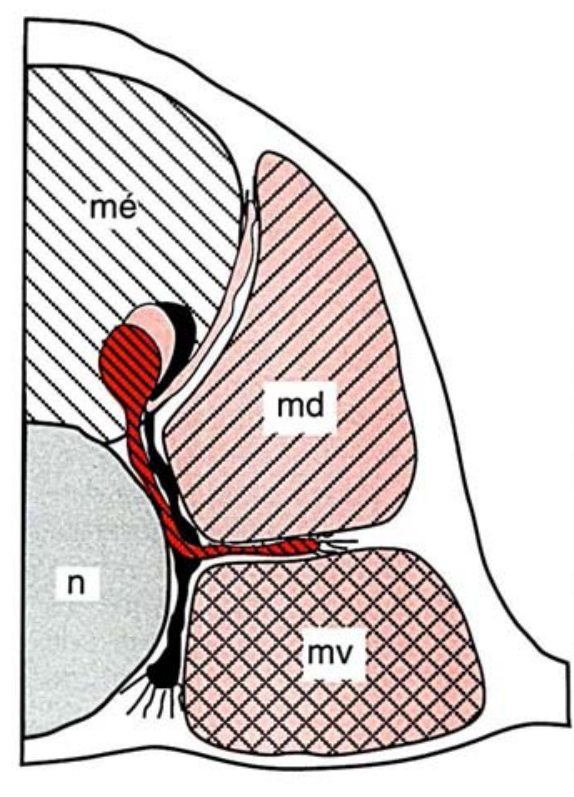

Figure 3. Croissance des motoneurones primaires innervant la musculature du tronc chez l'embryon de danio. A. Vue latérale où la face dorsale se trouve en haut et la direction rostrale sur la gauche. CaP, MiP et RoP indiquent, respectivement, les motoneurones primaires caudal, intermédiaire et rostral. Le temps, en heures, après la fécondation, est indiqué à la partie supérieure de la figure. Le septum horizontal, où se séparent les cônes de croissance des axones, est indiqué par des traits. Échelle : le trait au bas du schéma représente $20 \mu \mathrm{m}$. B. Vue transversale 19 heures après la fécondation. mé : moelle épinière ; $n$ : notocorde ; md : muscles dorsaux ; mv : muscles ventraux. (Adaptée de [8, 9]).

des contacts qu'une cellule neuronale doit établir avec d'autres cellules afin de remplir sa fonction. La relative simplicité du système nerveux de l'embryon de danio, la possibilité d'identifier des cellules à un stade précoce de leur développement et de les marquer ont permis d'accroître notre connaissance du développement du système nerveux.

Les neurones du danio apparaissent en deux vagues successives. Ceux de la première vague, appelés neurones primaires, se distinguent par leur développement précoce et leur plus gros corps cellulaire. Ils sont en nombre plus restreint que ceux de la seconde vague, dits neurones secondaires [5]. Les neurones primaires sont les premiers à projeter un axone et forment les premiers faisceaux de fibres nerveuses [6] qui serviront de guides aux axones des neurones apparaissant ultérieurement [1]. Les deux types de neurones pourraient jouer des rôles distincts [5]. Le développement du système nerveux des embryons homozygotes pour la mutation ned-1 (pour neural degeneration) [7] semble normal durant la période d'apparition des neurones primaires. Cependant, au cours du deuxième jour de la vie embryonnaire, tous les types de neurones secondaires des mutants commencent à dégénérer alors que les neurones primaires ne semblent pas être affectés. Il semble donc que, en plus des critères morphologiques, les neurones primaires et secondaires diffèrent selon des critères d'expression génétique.

De nombreux neurones primaires ont été identifiés et caractérisés chez l'embryon du danio et chez l'adulte. Les trois ou quatre motoneurones primaires qui innervent chaque hémi- 
segment de la musculature du tronc ont reçu une attention particulière. Judith Eisen et ses collaborateurs ont pu identifier chacun de ces motoneurones primaires selon la position qu'occupent leurs corps cellulaires dans la moelle épinière ventrale et par la spécificité avec laquelle ils projettent leurs axones vers des régions distinctes de la musculature du tronc [8, 9] (figure 3). En marquant ces neurones avec des colorants non toxiques à un stade antérieur à la croissance de leur axone, J. Eisen et al. [8] ont démontré que les axones de ces motoneurones quittent la moelle épinière selon une séquence prédéterminée. Les cônes de croissance de ces axones sont guidés vers la région qu'ils doivent innerver et s'y dirigent sans commettre d'erreurs apparentes de navigation. Afin de mieux comprendre les facteurs qui influencent la croissance des axones des motoneurones, leur environnement a été perturbé et la réponse de ces cellules analysée. Le rôle des interactions entre un motoneurone et ses voisins, au sein d'un même segment, a été étudié en faisant l'ablation d'une de ces cellules par irradiation avec un rayon laser ([10] et références citées]. Le comportement des cellules restantes est observé et apparaît normal dans tous les cas, ce qui indique que chaque motoneurone peut accomplir son programme de croissance indépendamment de la présence des autres [11]. De plus, les expériences d'ablation d'un motoneurone dans un segment donné ont permis de montrer que les axones de ces neurones n'entrent pas en compétition pour l'innervation du muscle. Même si les cônes de croissance se dirigent invariablement vers la région appropriée du tronc, certaines branches sont parfois formées dans une zone inappropriée. Ces branches secondaires se rétractent par la suite et ce, même après l'ablation du neurone qui doit normalement innerver la région où se trouve la branche secondaire [12]. La rétraction des branches secondaires se trouvant en territoire non approprié ne résulte donc pas d'une compétition entre les motoneurones mais de la réponse différentielle de ces cellules à des facteurs se trouvant dans leur environnement. Le groupe de Judith Eisen $\mathrm{m} / \mathrm{s} n^{\circ} 6$, vol. 7 , juin-juillet 91 cherche actuellement à identifier ces facteurs soit en déplaçant des cellules musculaires ou nerveuses vers des positions anormales, soit par des expériences de transplantation de cellules entre des mutants (comme par exemple le mutant spt-1) (voir plus loin) et des individus normaux.

\section{Génétique du danio}

Les travaux de George Streisinger et de ses collègues ont posé les bases sur lesquelles la génétique du danio a été construite. Streisinger a mis au point la production de souches clonales de danio dont les individus sont totalement homozygotes et ne portent aucune mutation létale [13-14]. Les souches clonales fournissent un fond génétique homogène d'où l'on peut faire ressortir de nouvelles mutations [14-15]. La transparence de l'embryon du danio permet de détecter des mutations sur de simples critères morphologiques sans avoir à interrompre pour autant la croissance des embryons.

La recherche de nouvelles mutations est grandement facilitée par la possibilité de les mettre en évidence dans la descendance directe des individus porteurs de la mutation (génération $F_{1}$ ), soit en rendant les descendants haploïdes, soit en produisant des descendants diploïdes homozygotes par gynogenèse* [13]. La figure 4 , p. 559, décrit la stratégie utilisée actuellement par Charles Kimmel et ses collaborateurs pour la production de nouvelles lignées de mutants. Les embryons sont irradiés avec des rayons $\gamma$ à un stade précoce du développement et seules sont conservées les femelles qui ne présentent aucune anomalie visible. Les individus haploïdes sont viables, du moins tout au long du développement embryonnaire. Cette approche élimine la nécessité d'attendre la maturité sexuelle des poissons de la génération $F_{1}$ pour procéder à des croisements permettant d'obtenir des mutants homozygotes à la génération $F_{2}$. Une mutation produisant un phénotype létal peut être maintenue à l'état hétérozygote en croisant la mère porteuse avec un mâle de type sauvage (figure 4, p. 559).

\footnotetext{
* Gynogenèse : production d'individus diploïdes dérivés uniquement des gamètes fermelles.
}

L'irradiation aux rayons $\gamma$ s'est avérée un traitement efficace pour la production de nouvelles mutations. Environ $33 \%$ des femelles irradiées ayant conservé un phénotype normal produisent des descendants chez lesquels on peut détecter un phénotype mutant. Certaines de ces mutations semblent affecter un groupe particulier de cellules alors que d'autres ont des effets pléiotropiques. Nous nous limiterons ici à deux exemples : les mutations fub-1 et spadetail (spt-1).

Le développement des cellules des muscles striés est perturbé par la mutation fub-1 ainsi que par deux allèles non complémentaires de cette mutation [16]. Les embryons homozygotes pour cette mutation sont paralysés, les muscles squelettiques du tronc se caractérisent par leur absence de striation et les myofibrilles composant ces muscles sont désorganisées. Ces cellules musculaires se développent toutefois au bon endroit, adoptent une forme normale, sont correctement innervées et expriment les mêmes protéines contractiles que les cellules correspondantes d'un poisson de type sauvage. Adam Felsenfeld et ses collaborateurs ont créé des embryons de danio mosaïques en irradiant des individus hétérozygotes au locus fub- 1 avec des rayons $\gamma$ peu de temps après la fécondation (stade de 2 cellules). L'irradiation rend probablement une des cellules homozygote pour la mutation fub-1. Chez ces individus mosaïques, on retrouve des cellules musculaires présentant le phénotype mutant au milieu de cellules musculaires normales, ce qui suggère que la mutation agit de manière autonome au niveau de la cellule. La mutation fub-1 affecte donc probablement un gène dont le produit est nécessaire à l'organisation des myofibrilles des cellules des muscles striés.

Alors que la mutation fub-1 affecte un type particulier de cellules, la mutation spt-l est hautement pléiotropique [17]. Les individus mutants se caractérisent par la présence d'un amas de cellules non différenciées au bout de la queue prenant la forme d'une bêche (d'où le nom spadetail) et par de sévères anomalies de la segmentation du mésoderme au niveau du tronc. De plus, les motoneurones 


\section{RÉFÉRENCES}

21. Njølstad PR, Molven A, Arpold J Fjose A. The zebrafish homeobox gene hox 2.2 : transcription unit, potential regulatory regions and in situ localization of transcripts. EMBO J 1990 ; 9 : 515-24.

22. Robert B, Sassoon D, Jacq B, Gehring W, Buckingham M. Hox-7, a novel homeobox gene with a novel pattern of expression during embryogenesis. EMBO J $1989 ; 8$ : 91-100.

23. Hill RE, Jones PF, Rees AR, et al. A new family of mouse homeobox-containing genes : molecular structure, chromosomal location, and developmental expression of Hox-7.1. Genes Dev 1989; 3 : 26-37.

24. Stuart GW, McMurray JV, Westerfield M. Replication, integration, and stable germ-line transmission of foreign sequences injected into early zebrafish embryos. Development $1988 ; 103: 403-12$.

25. Stuart GW, Vielkind JR, McMurray JV, Westerfield M. Stable lines of transgenic zebrafish exhibit reproducible patterns of transgene expression. Development 1990 ; $109: 577-84$

26. Westerfield M. Does the mesoderm induce hox gene expression in the zebrafish nervous system? Soc Neurosci Abstr 1990 ; $16: 302$.

27. Chakrabarti S, Streisinger G, Singer F, Walker C. Frequency of $\gamma$-ray induced specific locus and recessive lethal mutations in mature germ cells of the zebrafish, Brachy danio rerio. Genetics 1983 ; 103 : 109-23.

28. Capecchi MR. The new mouse genetics : altering the genome by gene targetting. Trends Genet $1989 ; 5: 70-6$.

29. Kimmel CB. Genetics and early development of zebrafish. Trends Genet $1989 ; 5$ : primaires innervant la musculature de cette région se développent anormalement. En suivant la migration de cellules marquées chez le mutant, Charles Kimmel et ses collaborateurs ont démontré que les cellules destinées à contribuer au mésoderme du tronc migrent anormalement au cours de la gastrulation et se dirigent plutôt vers les rudiments de la queue où elles adoptent diverses voies de différenciation ou bien demeurent à l'état non différencié et dégénèrent. Après avoir marqué et transplanté des cellules provenant de mutants spadetail chez des individus sauvages, Robert Ho et Judith Eisen ont observé que les précurseurs des cellules musculaires se dirigent toujours vers la queue lorsqu'ils sont placés dans un embryon normal, ce qui suggère que la mutation agit de manière autonome au niveau de ces cellules [18, 19]. En revanche, lorsque des motoneurones dérivés de mutants spadetail sont transplantés vers un individu de type sauvage, ils se développent alors normalement. Il semble donc que ces neurones répondent au produit du gène affecté chez le mutant spadetail mais n'aient pas besoin d'exprimer ce gène eux-mêmes.

\section{La génétique moléculaire du danio}

L'analyse moléculaire des gènes impliqués dans les processus de développement a été récemment entreprise dans quelques laboratoires dont celui de Monte Westerfield à Eugene et celui d'Anders Fjose en Norvège. De plus, la mise au point, par Gary Stuart et Monte Westerfield, d'une technologie permettant la production de danio transgéniques permettra, en manipulant ces gènes, de mieux comprendre leur rôle au cours du développement.

Parmi les gènes isolés chez le danio et qui jouent potentiellement un rôle au cours de l'embryogenèse, citons : deux gènes apparentés au gène engrailed de la drosophile ([20] et Akimenko et al., résultats non publiés) ; plus d'une demi-douzaine de gènes ([21] et références citées) possédant une boîte homéo de la même famille que celle d'Antennapedia, un autre gène impliqué dans le développement de la mouche ; trois gènes apparen- tés au gène Hox-7 murin ([22, 23] et Ekker et al., résultats non publiés) ; des gènes homologues aux gènes wnt-1 (précédemment nommé int-1) et $T$ de la souris (communications personnelles de, respectivement, Anders Molven et Stefan Schulte-Merker). Tous ces gènes sont exprimés à des stades spécifiques de l'embryogenèse et dans des régions spécifiques de l'embryon. Il ressort de leur caractérisation une grande similitude entre les gènes du danio et ceux d'autres espèces de vertébrés tant au niveau de la séquence en acides aminés prédite pour leur produit qu'au niveau de la distribution de leurs ARN messagers chez l'embryon. Cela suggère que ces gènes pourraient être appelés à jouer des rôles similaires au cours du développement d'espèces très éloignées et, donc, qu'un grand nombre de stratégies de développement auraient été conservées au cours de l'évolution. L'utilisation du danio en tant que modèle pour la manipulation de ces gènes et/ou la création de mutants devrait fournir des informations facilement transposables à d'autres espèces.

L'introduction de fragments d'ADN clonés dans un embryon, leur intégration dans le génome et leur transmission à la descendance s'avèrent non seulement utiles à l'étude fonctionnelle de produits de gènes mais constituent également un moyen de créer de nouvelles mutations par insertion. Des lignées de poissons transgéniques ont été produites par micro-injection dans le cytoplasme de l'œuf de danio, immédiatement après la fécondation, de constructions contenant le gène codant pour une enzyme bactérienne, la chloramphénicol acétyltransférase, utilisé comme marqueur, placé sous le contrôle de divers promoteurs viraux. Gary Stuart et al. [24, 25] ont montré que de telles constructions étaient intégrées au niveau du génome et transmises à la descendance avec une efficacité globale de $5 \%$. Ce taux est plus bas que ce que l'on obtient chez la souris à cause du haut degré de mosaïcisme chez le poisson. En revanche, la micro-injection est considérablement plus facile chez le danio et par conséquent un plus grand nombre de poissons transgéniques peuvent être produits au cours d'une 
session de travail. Le transgène est exprimé chez $18 \%$ des individus injectés $\left(\mathrm{F}_{0}\right)$ atteignant l'âge adulte et les poissons transgéniques des générations suivantes expriment le transgène selon un profil d'expression reproductible.

Une conséquence avantageuse du développement rapide de l'embryon de danio est la possibilité d'utiliser l'embryon entier comme système pour l'expression transitoire de transgènes. Dans ce cas, le transgène n'a pas besoin d'être intégré dans le génome pour fournir l'information recherchée. Monte Westerfield [26] a utilisé cette approche pour étudier l'expression de constructions conte-

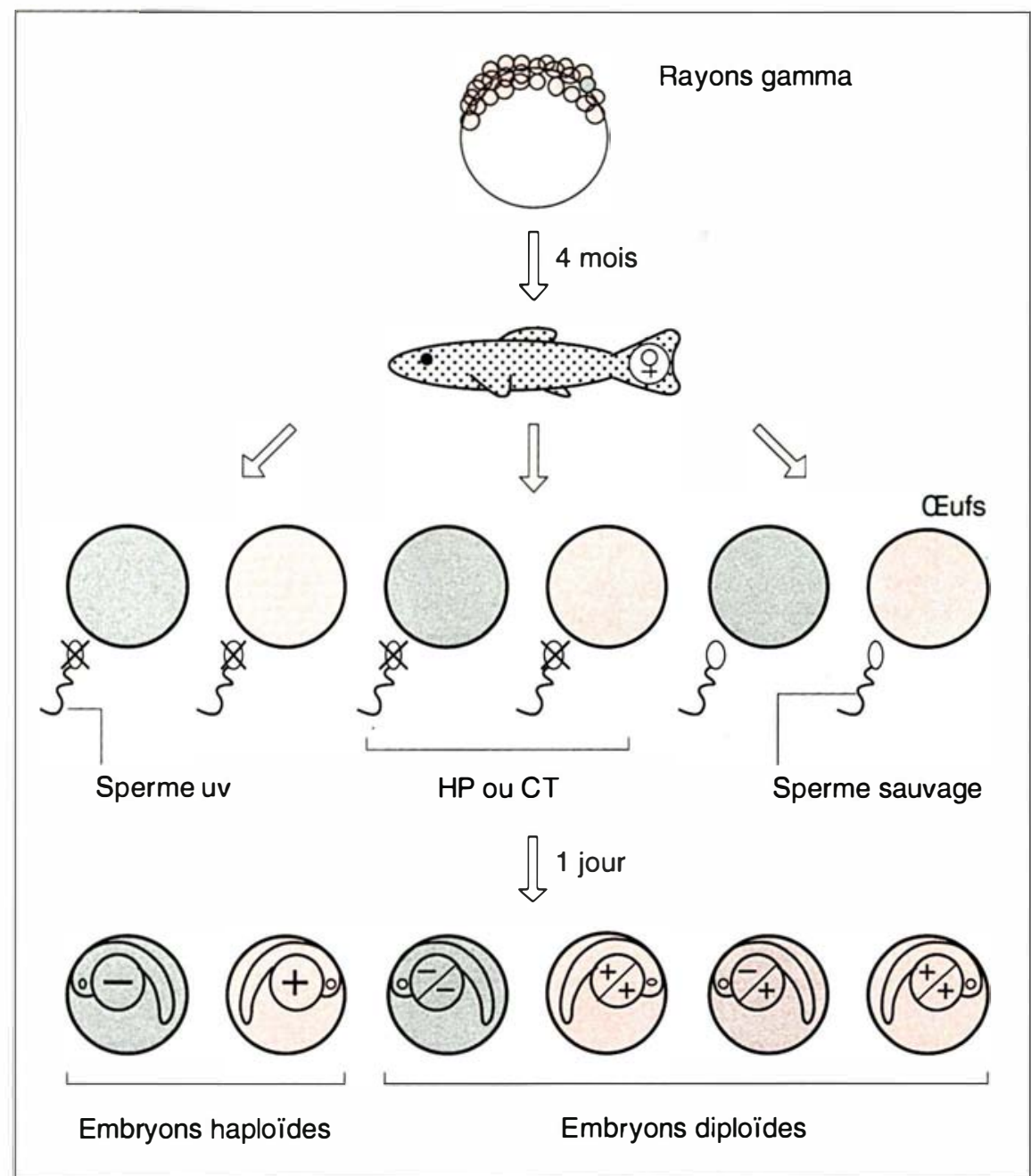

Figure 4. Stratégie pour la production et la conservation de nouvelles mutations. L'irradiation aux rayons $\gamma$ est un moyen de produire des mutations récessives dont l'effet peut être observé chez la descendance haploïde ou homozygote diploïde des femelles ayant été irradiées. Les embryons haploïdes sont produits en activant la division des œufs par des spermatozoides dont l'information génétique a été détruite par un traitement aux rayons ultraviolets (UV). Pour la production d'embryons diploïdes homozygotes, l'activation par les spermatozoïdes traités aux UV est suivie soit aussitôt par un traitement à haute pression (HP), soit par un choc thermique (CT) appliqué au cours du premier cycle cellulaire. Le traitement haute pression bloque la seconde division méiotique et produit un pourcentage de loci homozygotes qui dépend des événements de recombinaison qui ont eu lieu au cours de la première division méiotique. Le choc thermique bloque la première division mitotique et produit des embryons totalement homozygotes. Les mutations qui causent un phénotype létal peuvent être maintenues à l'état hétérozygote par fécondation des œufs avec des spermatozoides provenant de poissons de type sauvage. (Adaptée de [29].)

$\mathrm{m} / \mathrm{s} n^{\circ} 6$, vol. 7 , juin-juillet 91 nant les régions régulatrices de deux gènes à boîte homéo de mammifères, Hox 1.1 et Hox 3.3, liées à la séquence codante pour l'enzyme $\beta$ galactosidase dont on peut détecter l'activité à l'aide d'un substrat chromogène. Ces constructions sont exprimées chez l'embryon selon une distribution équivalente à celle que l'on observe chez la souris. L'expression apparaît en fin de gastrulation dans des cellules se dirigeant vers la partie postérieure de l'axe embryonnaire. Ultérieurement, l'expression est détectée dans les somites et la moelle épinière au niveau du tronc. Le profil d'expression est affecté chez les individus homozygotes pour la mutation spt-1 (voir plus haut) et se retrouve alors dans les mêmes tissus mais au niveau de la queue, région où les cellules mésodermiques devant contribuer au tronc aboutissent par erreur. Cette étude de l'expression transitoire de transgènes suggère qu'une induction du neuroectoderme par le mésoderme est impliquée dans l'expression de ces gènes à boîte homéo et que les gènes de développement montrent un haut degré de conservation entre les poissons et les mammifères, tant du point de vue de leur région codante que du point de vue des séquences impliquées dans le contrôle de leur expression.

\section{Perspectives et conclusion}

Une exploitation optimale du potentiel qu'offre le danio en tant que modèle pour une étude génétique du développement nécessite la disponibilité de quelques outils encore manquants. Le premier d'entre eux est une carte génétique des 25 chromosomes du danio. Cette carte peut être construite à l'aide de marqueurs, qui sont le plus souvent des séquences d'ADN anonymes pour lesquelles un polymorphisme de fragments de restriction a été observé. De nouvelles techniques qui facilitent la production d'une telle carte ont été récemment mises au point et d'autres le seront sans doute dans un avenir proche grâce à l'impulsion donnée par le projet de cartographie et de séquence du génome humain. La carte chromosomique du génome du danio, qui a environ la même taille que celui de l'homme ou de la souris, sera utile 
pour la caractérisation des régions chromosomiques affectées dans les expériences de mutagenèse aux rayons $\gamma$ décrites plus haut. Dans ces expériences, jusqu'à $1 \%$ du génome peut être perturbé [27].

Deux autres outils essentiels à une approche génétique du développement sont, d'une part, la possibilité de provoquer des mutations par insertion et, d'autre part, celle de muter sélectivement un gène donné. La mutagénèse par insertion permet de créer de nouveaux phénotypes et d'identifier les gènes impliqués. En effet, le gène associé au phénotype mutant peut être cloné en se servant des séquences connues qui s'y sont insérées. Chez la drosophile, la présence d'éléments transposables facilite la mutagenèse par insertion. Dans le cas du danio, il faudra soit identifier d'éventuels éléments transposables, soit augmenter le taux d'insertion des transgènes de manière significative afin de rendre possible cette approche.

L'inactivation sélective d'un gène a été récemment rendue possible chez la souris grâce à la technique de recombinaison homologue dans des cellules souches embryonnaires pluripotentes [28]. Cette approche apportera sans doute une information importante quant au rôle de plusieurs gènes que l'on soupçonne être impliqués dans les processus de développement. La mise au point d'une telle technique chez le danio serait sans doute d'un grand intérêt, mais sa faisabilité reste encore à démontrer.

Une combinaison d'approches embryologiques et génétiques est nécessaire à notre compréhension des mécanismes de développement chez les vertébrés. Le danio rerio, qui permet déjà une approche embryologique, peut aussi se prêter à une approche génétique. Lorsque celle-ci aura été développée, ce petit poisson constituera un modèle des plus importants pour l'étude du développement. Un grand nombre de stratégies du développement semblant avoir été bien conservées au cours de l'évolution, les connaissances acquises par l'étude du danio ouvriront la voie à la compréhension de la biologie du développement chez d'autres espèces de vertébrés, dont la nôtre

\section{Summary}

Embryology and genetics of the zebrafish, Brachydanio rerio

A combination of approaches in embryology and genetics is necessary for our understanding of vertebrate development. The zebrafish is a simple vertebrate with many features that facilitate the study of development. Embryos are easily accessible at all stages and can be produced in large numbers. They develop rapidly ; embryos hatch at two days, and most morphological features of the adult are visible at seven days. Most importantly, genetic methods developed to obtain haploid or gynogenic offsprings and lines of transgenic animals provide additional advantages for using zebrafish for the study of development. These methods are summarized here, along with a few studies in which the zebrafish is used as a model to address questions such as embryonic cell fate determination and the specificity of motoneural connection. These studies emphasize the importance of the availability of mutant embryos in which specific aspects of development are perturbed. Finally, a high degree of conservation exists between the zebrafish and distantly related vertebrate species in genes that are thought to be involved in developmental processes. This enhances the attractiveness of the zebrafish as a simple model of our own development.

\section{TIRÉS A PART}

\section{Ekker.}

\section{Remerciements}

Nous remercions Claire Bonnerot, Pascale Doutriaux et Georges Lutfalla pour les commentaires qu'ils ont apportés suite à la lecture de ce manuscrit. Nous remercions également les membres des laboratoires "danio " d'Eugene pour les photographies de la figure 1. M.E. est titulaire d'une "Bourse du Centenaire "du Conseil de Recherches Médicales du Canada. M.-A. A. bénéficie de l'appui de l'Institut Pas teur de Paris et d'une bourse de l'OTAN. 